\title{
Implementation of a Web-based remote control system for qZS DAB application using low-cost ARM platform
}

\author{
M. KORZENIEWSKI ${ }^{1 *}$, K. KULIKOWSKI ${ }^{1}$, J. ZAKIS ${ }^{2}$, M. JASINSKI ${ }^{3}$, and A. MALINOWSKI ${ }^{4}$ \\ ${ }^{1}$ Department of Power Electronics and Electric Drives, Bialystok University of Technology, 45 D Wiejska St., 15-351 Białystok, Poland \\ ${ }^{2}$ Department of Electrical Drives and Power Electronics, Tallinn University of Technology, 5 Ehitajate St., 19086 Tallinn, Estonia \\ ${ }^{3}$ Institute of Control and Industrial Electronics, Warsaw University of Technology, 1 Politechniki Sq., 00-661 Warsaw, Poland \\ ${ }^{4}$ Department of Electrical and Computer Engineering, Bradley University, Peoria, IL 61625, USA
}

\begin{abstract}
Continuous development of intelligent network applications drives the demand for deployment-ready hardware and software solutions. Such solutions are highly valued not only by distributed producers of energy but by energy consumers as well. The use of intelligent network applications enables the development and improvement of the quality of services. It also increases self-sufficiency and efficiency. This paper describes an example of such device that allows for the control of a dual active bridge (DAB) converter and enables its remote control in real time over an IP-based network. The details of both hardware and software components of proposed implementation are provided. The DAB converter gives a possibility to control and manage the energy between two DC power systems with very different voltage levels. Not only information, but also the quality of energy, the direction of power flow, and energy storage systems can be easily controlled through an IP-based network and power electronics converters. Information technology, together with intelligent control of power electronics technology, provides a flexible solution, especially for sustainable smart grids.
\end{abstract}

Key words: ARM processor, DAB converter controller, networked controller, Web-based user interface, energy control.

\section{Introduction}

The technology of information and energy processing advances significantly every year. The energy conversion process can be precisely controlled using both classic, and artificial intelligence-based algorithms [1-3], thanks to powerful microcontrollers. Moreover, modern platforms assure the possibility of real-time operation, interaction, data processing, and prediction [4]. New semiconductor materials, such as silicon carbide and gallium nitride [5], make it possible to reduce power losses and improve the compactness of power electronics devices [6]. Different topologies of power electronics converters assure flexible energy conversion, both AC-DC and DC-DC, like dual active bridge $(\mathrm{DAB})$, with a requirement thereof in future sustainable AC power systems [7], and most recent DC power systems [8].

At the same time, the development in the areas of electrical energy storage and contactless power transmission opens new possibilities for control and power flow management $[9,10]$. Hence, these possibilities should be taken into account in the context of an electrical smart grid, where the significance of power electronics converters is growing. A good definition of a smart grid is provided by ABB [11]: "A smart grid is an evolved grid system that manages electricity demand in a sustainable, reliable and economic manner, built on advanced

*e-mail: m.korzeniewski@pb.edu.pl infrastructure and tuned to facilitate the integration of all involved". It became truly multidisciplinary, as it attracts research and development not only by electrical engineers, but also by entrepreneurs, economists, sociologists, politicians, lawyers, and finally, IT-specialists $[12,13]$. However, a smart grid is not only smart metering, but also smart controlling of the energy conversion process and power flow management (flow direction, energy storage and conditioning). The two most recent decades were dedicated to Internet communication. The next two or more decades will not be focused only on the Internet, but also on the Energy Net network, where it is the energy that will be managed, instead of information [14]. The tools necessary to implement this vision are microchips like digital signal processors (DSPs), complex programmable logic devices (CPLDs), field programmable gate arrays (FPGAs) or advanced RISC machines (ARMs) to, in real time, control transistors and thyristors switching in different types of power electronics converters.

Because the technology of reduced instruction set computer (RISC) is flexible and achieved a sufficient level of maturity, the price of ARM devices became very competitive. That leverages the ARM platform as a suitable tool to handle intelligence communication and at the same time provide robust operation at a reasonable price [15]. This ARM-based solution provides the environment for real-time communication using different protocols.

The paper is focused on an ARM-based solution for the control and Web-based management of a DC-DC power elec- 
tronic converter in the topology of qZS DAB [16, 17]. This topology is very challenging from the point of view of flexible interface converter in smart grid applications. It can provide bidirectional power flow among the power source, power grid, and storage. Due to the input voltage boost capability in both directions, the proposed converter is an effective power conversion tool and can reveal all benefits of the proposed control system. The proposed control system is optimized for a highly demanding application where ten switches ( 8 transistors and 2 shunting switches) should be controlled. Moreover, the unconventional switching sequence can be performed in order to achieve zero voltage switching (ZVS) or zero current switching (ZCS) [18].

Hence, it can be treated as an interface between two different DC power systems, or between a DC power system and an energy storage device. DAB can be used in wide power range applications.

In the Internet age (and in the coming Energynet) the Webbased management capability of a device is important from the prosumers' point of view. Each prosumer can interact through a user-friendly interface with energy data acquired in its power system (like smart srids or smart industry). The subject of the smart grid has become an area of research for a number of scientists and resulted in an abundance of publications. Many of those publications focus on showing the idea of the communication and control link between a power electronics converter and the prosumer as shown in Fig. 1.

The remainder of this paper is organized in the following way. Section 2 describes the hardware platform of the controller board including an ARM-based microcontroller and peripheral devices. Section 3 describes the DAB used for this application. Section 4 describes the firmware, including the communication framework with TCP/IP stack, the control algorithm, and the

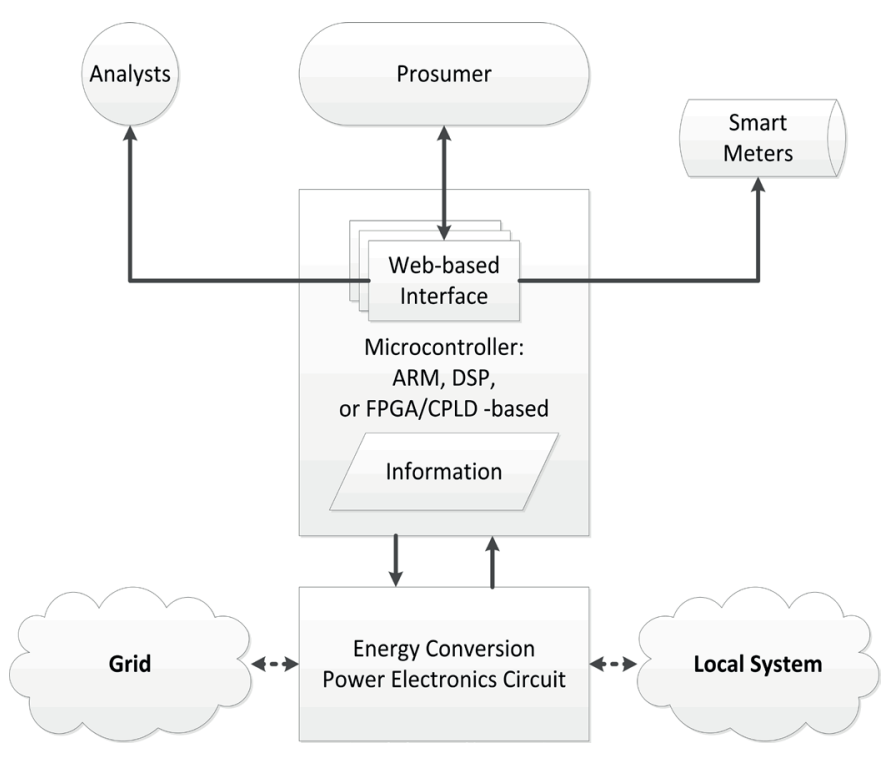

Fig. 1. The idea of ARM-based communication and control link between power electronics converter (energy conversion) and prosumer
Web-based diagnostic and control interface. Section 5 demonstrates the laboratory tests of the manufactured controller. The conclusions are presented in Section 6.

This work provides an example of design, and describes the principles of operation of a controller for one of such devices - the, in author's opinion, very promising power converter qZS DAB unit.

As far as authors know, the novelty of this paper lays in the proposition of complex solution for qZS DAB converter monitoring and control through the Internet in real time. A dedicated control board, designed specifically to fulfill all the mentioned requirements, has been developed and integrated with the constructed efficient qZS-DAB converter. Software for control and data transfer has been developed and implemented. The whole system has been tested in laboratory environment.

\section{Inverter control system}

2.1. Main control unit. The control system is based on a 32-bit ARM Cortex-M4 microcontroller from ST Microelectronics (ST). The ARM Cortex-M is a group of 32-bit RISC ARM processor cores licensed by ARM Holdings. The cores are intended for use as microcontrollers and they include the Cortex-M0, Cortex-M1, Cortex-M3, Cortex-M4 and Cortex-M7 [19]. Internally, each microcontroller consists of the processor core, static RAM memory, flash memory, debugging interface, and various configuration-dependent peripherals. The ARM core designs have numerous configurable options, and ST choose to use an individual custom configuration for each design. ST attach their own peripherals to the core before implementing the design in a silicon die.

The ARM Cortex ${ }^{\mathrm{TM}}-\mathrm{M} 4$ processor is the most popular choice for embedded applications, and was developed specifically to address digital signal control markets that demand an efficient, easy-to-use blend of control and signal processing capabilities. The Cortex-M4F (STM32F407) processor features extended single-cycle multiply-accumulate instructions, optimized SIMD arithmetic, saturating arithmetic instructions and an optional single-precision floating point unit (FPU). These features build upon the innovative technology that characterizes the ARM Cortex-M processor family.

The combination of high-efficiency signal processing functionality with the low-power, low-cost and ease-of-use benefits of the Cortex-M family of processors is designed to satisfy the emerging category of flexible solutions, targeting specifically motor control, automotive applications, power management, embedded audio and industrial automation markets [20].

The STM32F4 series consists of five compatible product lines of digital signal controllers (DSC), all being a perfect blend of the real-time control capabilities of an MCU and the signal processing performance of a DSP. The STM32F4 is perfectly suited for DC and AC three-phase machine control with a wide range of products. Additionally, it contains an embedded ethernet media access control with dedicated DMA and IEEE 1588 precision time protocol hardware support. 
2.2. Hardware configuration of the control system. The block diagram of the control system is shown in Fig. 2. The common 4-layer board of the control system with the microcontroller contains connectors such as SWD programmer, LCD console, Ethernet, USB, analog inputs and SPI serial interface. This system was designed and developed to control power electronic converters (AC-DC, DC-DC and DC-AC) via fiber-optic lines or wiring. The main microcontroller can run with clock speeds up to $168 \mathrm{MHz}$ and comes with optional built-in hardware for Ethernet support. However, only select few models from the MCU family, that is STM32F7, STM32F4, STM32F3, and STM32F1 series, provide Ethernet support as included. Ethernet tends to be one of the exclusive features in the special series MCUs and in newer and more expensive devices. They utilize the reduced [standard] media independent interface (RMII) protocol (IEEE 802.3u) using a DP83848 transceiver chip. That allows for both 10 and $100 \mathrm{Mb} / \mathrm{s}$ transmission speeds. The use of RMII, rather than standard MII allows for reduction of the number of $\mathrm{I} / \mathrm{O}$ pins used for communication between the Ethernet transceiver and media access control (MAC) hardware. Originally, it was intended mostly for devices with multiple Ethernet ports, such as switches, to reduce the number of PINS and routed connections on a PCB.

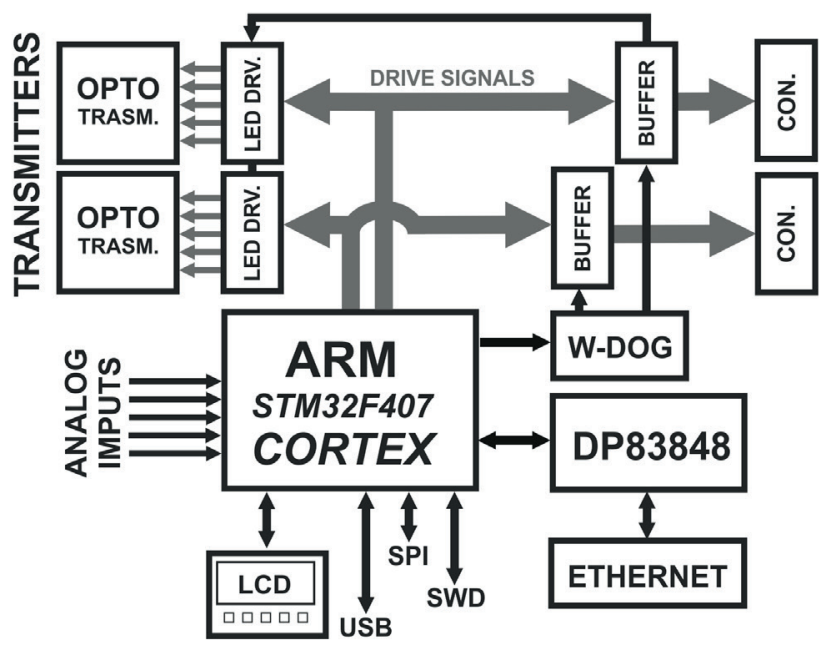

Fig. 2. Block diagram of the inverter control part

The DP83848C from Texas Instruments is a robust, fully featured 10/100 single port Physical Layer device that receives the 4-bit wide RMII protocol, and synthesizes the differential signals necessary to drive the Ethernet RJ45 socket. It also does the reverse, decoding incoming signals from the RJ45 connector and talking RMII back to the MCU.

The electrical interface contains a 1:1 isolation transformer between the outside connection and the physical layer (PHY). The PCB board shown in Fig. 3 is equipped with a six-channel analog input connector, used for measurement of currents and voltages of the inverter utilizing a 12-bit A/D converter. An
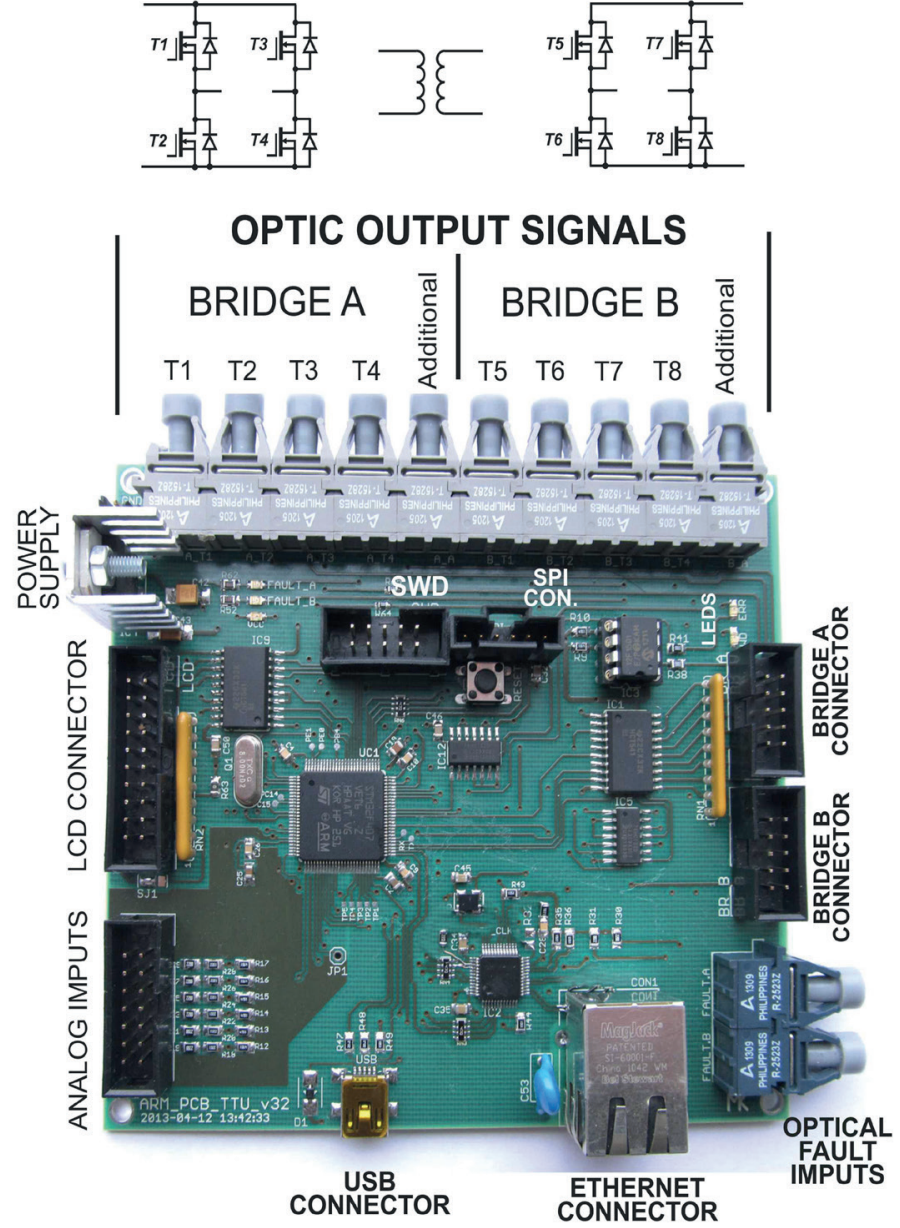

Fig. 3. Top view of the designed and developed control board circuit with connector descriptions in a typical application of a DC-DC converter

LCD connector allows for connecting an optional local LCD display, equipped with push buttons that may be utilized by software as a user interface, allowing to display the current status or to change the settings of the inverter parameters.

Programming and debugging of the MCU can be realized by using the Serial Wire Debug (SWD) interface. SWD replaces the JTAG interface commonly used in ARM7/9 and other microcontroller products. The SPI interface allows connecting external components, such as DACs or flash memory, or can be used to communicate without its control systems. An external watchdog chip installed on the PCB supervises the proper operation of the MCU. Unlike a built-in watchdog that would reset the microcontroller in case of malfunction, this external watchdog can disable the output signals to the converter.

The frequency of the PWM output signals can be adjusted within the range from 0 to $200 \mathrm{kHz}$ with 16-bit resolution. All these features, in combination with a single, precision floating point unit (FPU), enable implementation of complex control algorithms in real time, and at the same time, utilize communication via Ethernet using the low-cost ARM technology. 


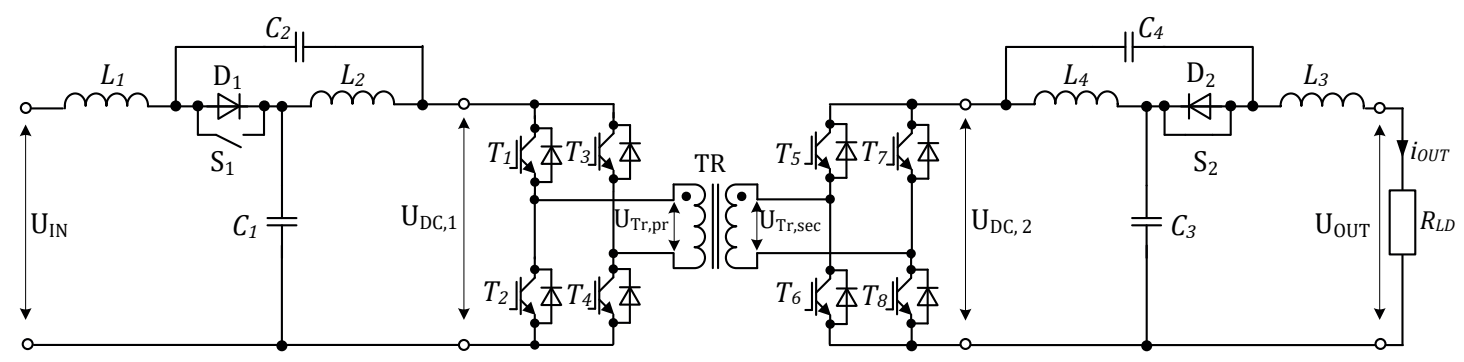

Fig. 4. General circuit diagram of qZS-based dual active bridge

\section{Hardware configuration of qZS DAB}

The experimental verification of the developed control board was carried out using quasi-Z-source (qZS) based dual active bridge (DAB) [21]. Fig. 4 presents the general circuit diagram of the qZS-based DAB used for the experimental verification.

The proposed DC/DC converter employs two distinct operating modes: forward and reverse. However, in the case of $\mathrm{DAB}$, the transistor pairs on both bridges are controlled using a phase-shifted PWM with a determined duty cycle of $\mathrm{D}=0.5$, whereas the qZS network performs the low-pass filtering. Normal operating conditions of the converter presume that the voltage levels of the two sources are at the desired level, and thus the sign of the phase angle $\varphi$ determines the power flow direction, and the angle value determines the magnitude of the transferred power.

Since the circuit is fully symmetrical, it can operate in both directions. The experiments were carried out in two operation modes: normal mode and boost mode. Since the diode $\mathrm{D}_{2}$ in this configuration (Fig. 4) is reverse biased, it is bypassed by the mechanical switch $\mathrm{S}_{2}$. For simplicity reasons, the load resistance $\left(\mathrm{R}_{\mathrm{LD}}\right)$ is used at the secondary side of the discussed converter.

a)

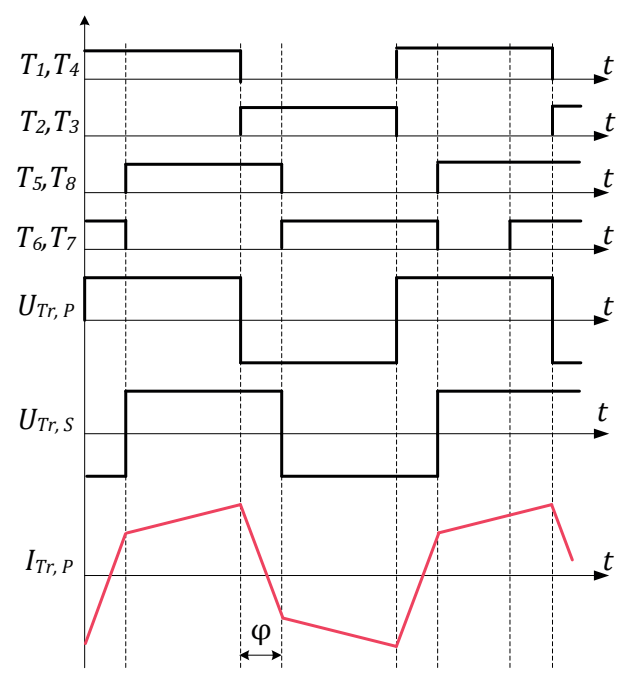

In normal operation mode, the energy is transferred from the primary side towards the secondary side. The transistors $\mathrm{T}_{1} \ldots \mathrm{T}_{4}$ on the primary side and $\mathrm{T}_{5} \ldots \mathrm{T}_{8}$ on the secondary side are switched in pairs, using the positive phase shift angle $\varphi$, as shown in Fig. 5b. In this operation mode, when the transistors of the inverter are working with maximal possible duty cycle $(\mathrm{D}=0.5)$, the input voltage should, theoretically, be equal to output voltage $\left(\mathrm{U}_{\mathrm{IN}}=\mathrm{U}_{\mathrm{OUT}}\right)$, neglecting the voltage drop on components. The regulation of power flow is achieved by regulating the phase shift angle $\varphi$.

However, during the operation, the voltage of the source $\left(\mathrm{U}_{\mathrm{IN}}\right)$ might decrease below the desired level. In such cases, the voltage boost properties of the qZS network must be utilized [25] to step-up the DC-link voltage $\left(\mathrm{U}_{\mathrm{DC} 1}\right)$ to the required level.

To boost up the voltage, the shoot-through operation mode should be introduced $[25,26]$. The shoot-through by the overlapping active states has the most optimal operating parameters [27], and it is for this reason why such a method was selected for the following analysis.

The output power of the converter can thus be controlled by the duration of the shoot-through state. In this case, an additional phase shift $\varphi$ is added to the gate signals of the HV side signals, to set the transformer current to a new level during the b)

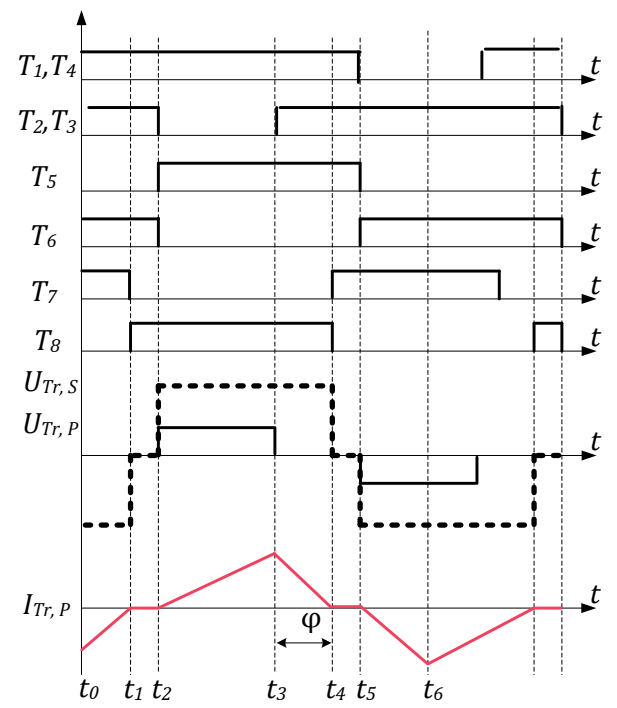

Fig. 5. Gate signals and the corresponding transformer voltages and current of qZS-based dual active bridge in: a) Normal mode, b) Boost mode 
a)

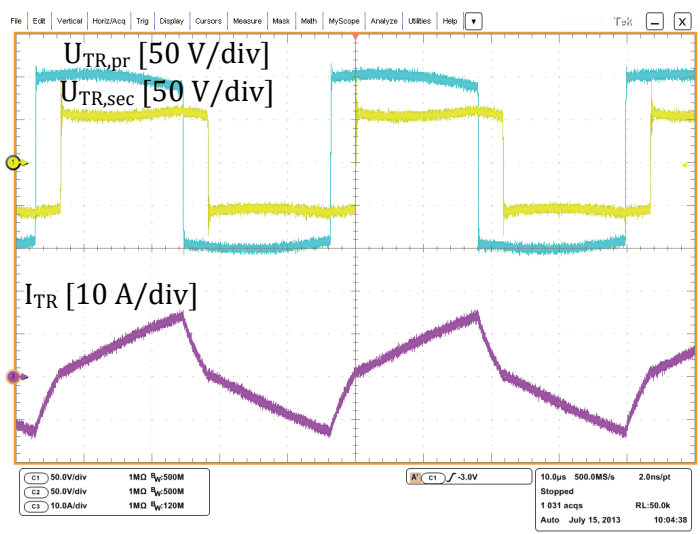

b)

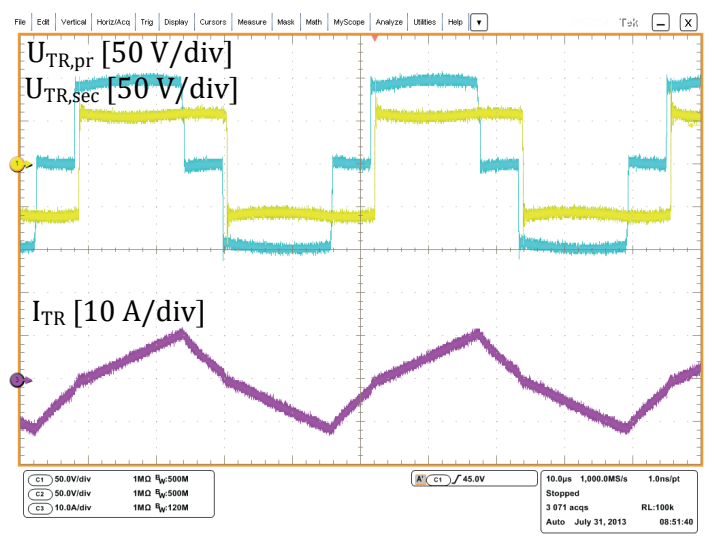

Fig. 6. Basic operation waveforms $\left(\mathrm{U}_{\mathrm{TR}, \mathrm{pr}}, \mathrm{U}_{\mathrm{TR}}\right.$, sec and $\left.\mathrm{I}_{\mathrm{TR}}\right)$ of a qZSI-based dual active bridge in normal operation mode: a) in Normal mode, b) in Boost mode

shoot-through state of the LV side transistor. However, in the proposed case, the phase shift duration is limited by the shootthrough state duration, thus using the phase shift that, in that case, has a limited power flow control capability.

During experiments, the input voltage is set at nominal level $(100 \mathrm{~V})$ according to Table 1 . The output voltage, in this case, should be almost the same ( $95 \mathrm{~V})$, including the voltage drop on components. In this operation mode, the transistor switches of both inverters $\left(\left(\mathrm{T}_{1}-\mathrm{T}_{4}\right)\right.$ and $\left.\left(\mathrm{T}_{5}-\mathrm{T}_{8}\right)\right)$ are switched in pairs using the positive phase shift angle $\varphi$. The inverter switches of primary side $\left(\mathrm{T}_{1}-\mathrm{T}_{4}\right)$ in normal mode works as a conventional VSI.

Fig. 6 a presents basic operation waveforms of the qZS-based dual active bridge in normal mode. It can be seen that the transformer primary voltage is shifted in relation to the secondary voltage by angle $\varphi$. The difference in primary and secondary voltage levels could be explained by the fact that in the secondary side, the load resistance is used. Also, it could be caused by a voltage drop on components. Generally, the experimental waveforms (Fig. 5a) are in good agreement with the theoretical ones. In the experimental verification of Boost mode (Fig. 5b) the input voltage is half of the nominal voltage $(50 \mathrm{~V})$, according to Table 1. For twofold input voltage boost, the gate signals with duty cycle $\mathrm{D}=0.75$ were applied on the switches, resulting in a shoot-through duty cycle $\mathrm{D}_{\mathrm{S}}=0.25$.

Table 1

Operating points of the $\mathrm{DC} / \mathrm{DC}$ converter

\begin{tabular}{|c|c|c|c|c|c|c|}
\hline Mode & $\begin{array}{c}U_{I N(D C)} \\
{[\mathrm{V}]}\end{array}$ & $D_{S}$ & $D_{A}$ & $\begin{array}{c}f_{s w} \\
{[\mathrm{kHz}]}\end{array}$ & $\begin{array}{c}U_{\text {OUT }(D C)} \\
{[\mathrm{V}]}\end{array}$ & $\begin{array}{c}\text { Power } \\
{[\mathrm{W}]}\end{array}$ \\
\hline Normal & 100 & 0 & 1 & 23 & 95 & 500 \\
\hline Boost & 50 & 0.25 & 0.75 & 23 & 95 & 500 \\
\hline
\end{tabular}

Fig. 6 presents the basic operation waveforms which confirm the correct operation of the qZSI dual active bridge. In this case, the voltage difference between the primary and the secondary voltages of the transformer windings can be observed as well. This can be caused by load resistance or voltage drop on converter components.

\section{Software design}

4.1. Communication framework with TCP/IP stack. The microcontroller firmware was developed using CoIDE environment for ARM ${ }^{\circledR}$ Cortex $^{\mathrm{TM}}$-M4 core-based STM32F4. The code footprint is $165 \mathrm{kB}$, with $80 \%$ being used by Ethernet components. The firmware utilizes only $32 \%$ of memory, as this ARM contains $512 \mathrm{kB}$ of flash EPROM.

TCP/IP communication stack and HTTP application layer protocol are essential for the interoperability of networked devices [25-28]. STM32 is supported by numerous TCP/IP stacks, such as LwIP, $\mu$ IP, CMX-TCP/IP [29] and more. LwIP is just one of widely used open-source stacks, and it is supported by ST Microelectronics [30]. It can be used either as a standalone product, event-driven by network traffic, or in conjunction with a real-time operating system. STM provides reference design software for both options, with the latter bundled with FreeRTOS. The stack supports both IPv4 and IPv6, and can be configured for either a static or dynamic IP address. STM claims that its typical memory footprint is $35-40 \mathrm{kB}$ of ROM and $30 \mathrm{kB}$ of RAM that is mainly used for Ethernet and IP stack buffers. The amount of RAM used can be reduced by decreasing the number of buffers, which at the same time decreases the ability to handle the volume of simultaneous Internet communication.

Both UDP and TCP protocols can be used for intranet or Internet communication, with the latter being capable of optionally being encrypted using secure socket layer technology. The UDP protocol is stateless, and its use without TCP could significantly reduce the memory footprint. It can be used in proprietary communication protocols, or by building on simple network management protocol (SNMP). TFTP could be used to transfer larger chunks of data or to update the firmware. In order to use more complex application protocols, such as an HTTP-based Web server, the TCP protocol needs to be included which dramatically increases the demand for memory buffers. 
However, HTTP, being highly standardized, allows for plugand-play connectivity to the networked device, and for both inspecting the device status and reconfiguring its behavior, using standard tools such as a Web browser. The networked device can be accessed by software that interacts with a Web server using the same protocol. Thus, the same interface can be used by both humans and machines.

LwIP comes with optional Web server application layer service, and therefore, there is no need to write a custom one. Web pages can be stored in ROM and infused with dynamic information using HTML-SSI tags, described in subsection C, or dynamically created by code ran on demand, from within the Web server, as CGI scripts. The latter allows reading and handling data and commands sent back to the Web server.

If desired, the communication can be secured using Secure Socket Layer (SSL) protocol provided by PolarSSL library. Furthermore, several chips in STM32F217xx and STM32F417xx series contain hardware support that allows for the alleviation of the computational expense of use of SSL [31].

4.2. Software design of the controller. Setup was made for DAB [18] control. The firmware program allows for complex forming of the signal-controlling transistors. The DAB-state settings can be applied by using either the control panel with an LCD display and a keyboard, or a Web-based interface. The program is able to the change the following parameters, as illustrated in Fig. 7:

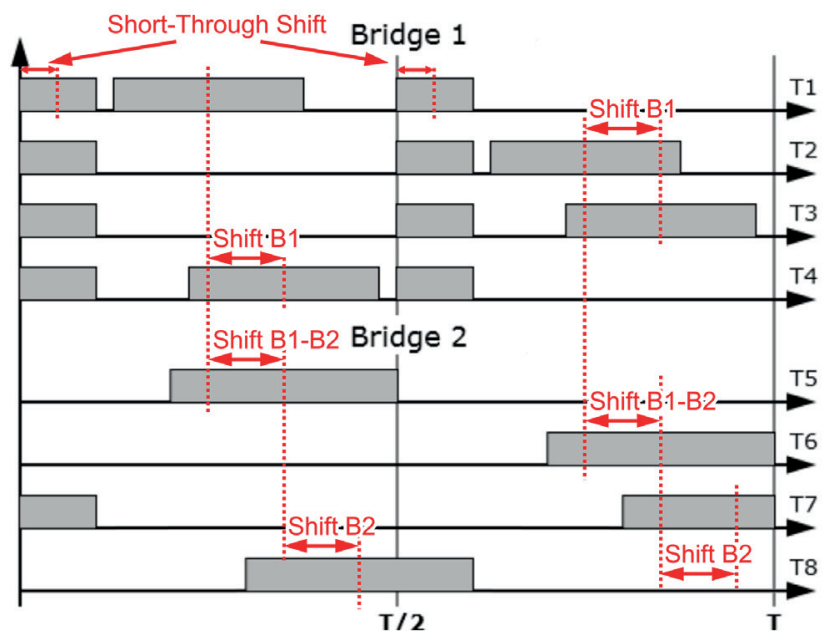

Fig. 7. Time shifting in $\mathrm{DAB}$ converter control signals

- The frequency of bridge 1 and bridge 2 transistors.

- Control signals, independently

- Frequency and duty cycle of additional signal control of bridge 1 (short-through)

- Time shift between legs of bridge 1 (shift B1)

- Time shift between legs of bridge 2 (shift B2)

- Time shift between bridge 1 and bridge 2 (shift B1-B2)

- Death time in bridge 2

- The duty cycle of each transistor independently

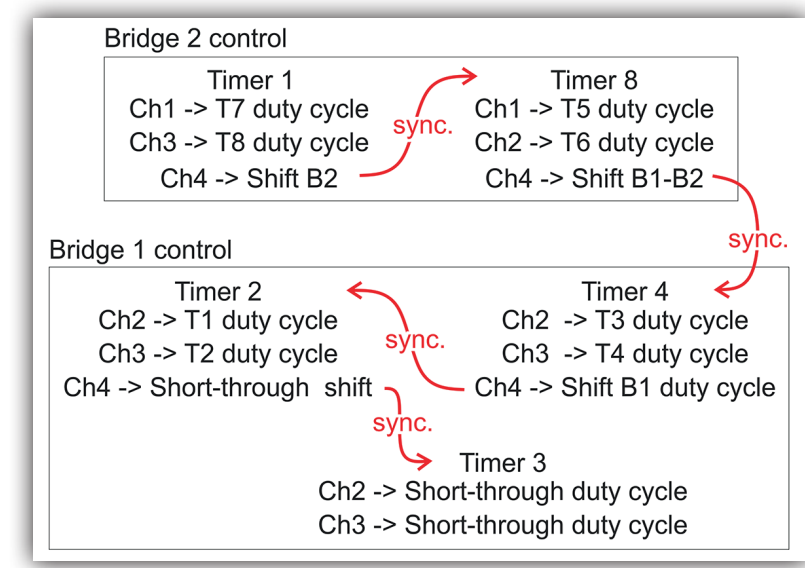

Fig. 8. Timer channels used in DAB control

Depending on chosen method, the tuning of some of the above parameters can be limited or completely disabled. The complex forming of transistor control signals was achieved by the use of five timers/PWMs, i.e. Timers 1, 2, 3, 4 and 8 as shown in Fig. 8. Timers 2, 3, and 4 control bridge 1. In a standard solution, the control of 4 transistors could be realized by a single timer. The additional Timer 4 is used to achieve flexible time-shifting between transistors T1->T4 and T2->T3. The use of Timer 3 enables flexible control of short-through signals, i.e. independent frequency and time shift (Fig. 7). Timers 1 and 8 control bridge 2 . Both timers have hardware dead-time control, as it was desired for the proposed solution.

The use of two timers allows for flexible control of time shifting between transistors T5->T8 and T6->T7. The time shifting is realized by proper synchronization of timers. Timer 1 is the main timer in the system control. Timer 8 is synchronized from channel 4 of Timer 1 that corresponds to the B2 shift (see. Fig. 8). Timer 4 is synchronized from channel 4 of Timer 8 (shift B1->B2). Timer 2 is synchronized from channel 4 of Timer 4 (shift B1). Timer 3 is synchronized from channel 4 of Timer 2 .

The program was designed to allow for very flexible configurations of the converter controller. However, that flexibility may be the cause of difficulties in choosing proper settings. In order to simplify the configuration process, a Web-based control interface was implemented. When using this interface, it is possible to verify the transistor signal settings before turning on the converter. From the computer network end, the controller is an Ethernet-connected device that runs a simple Web server. Any device connected to the same computer network, capable of running a JavaScript-compatible Web browser, enables handling the controller settings, via a user interface, as shown in Fig. 9.

4.3 Web-based control interface. In order to exchange data between firmware that implements the control algorithm and the Web server, the Server Side Includes (SSI) scripts are used. While sending the contents of a Web page, the server software checks whether a particular page includes SSI tags. If it does find proper tag, the server inserts additional, dynamically generated Web page code, based on the current settings of the controller. 


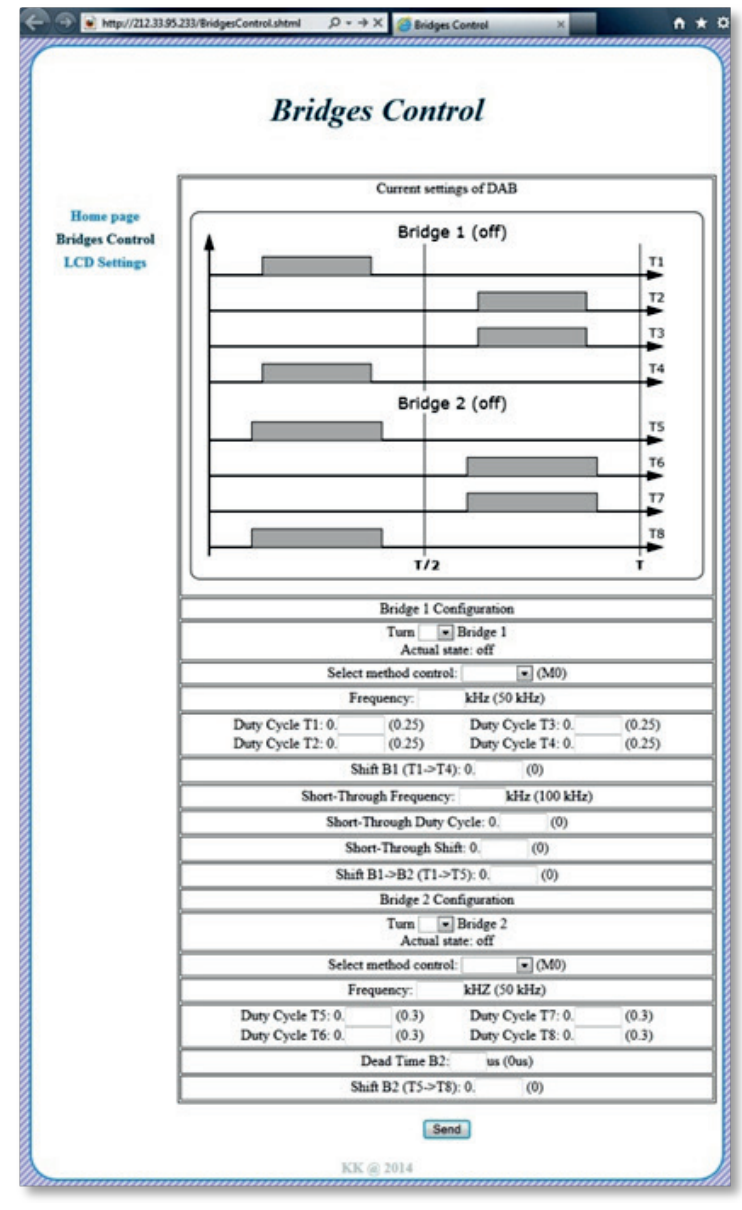

Fig. 9. Web page with converter bridges control settings

In our case, the tag name is " $<$ !--\#a---", and it is replaced by a JavaScript vector variable " $\mathrm{v}$ " of 21 values, describing the current bridge controller settings, as shown in Fig. 9.

That way the user always gets the current values of converter settings. The graphic interpretation of " $\mathrm{v}$ " vector is implemented in the JavaScript code portion of the Web page that is interpreted and executed by the Web browser as a program within the Web page, one that can modify page contents. Based on values encoded in the variable " $v$ ", the JavaScript code run inside the Web browser presents the current state of the converter settings in a graphical way. The web page contains a canvas layer (shown in Fig. 10) that allows JavaScript code to draw a graph, which is shown on that Web page after it finishes loading and is updated, as the settings are being changed by a user manipulating the contents of the form located below the graphics. That way, the settings of the transistor control signal can be easily verified before they are committed as actual settings, and before they are applied to the control algorithm. After they are visually verified and committed, the changes to bridge 1 and bridge 2 result in the controller producing the signals for transistors control, generated in exactly the same way that is visualized in the Web browser.

When the user commits the new settings, another Web page request is sent by the Web browser. This time, the request is

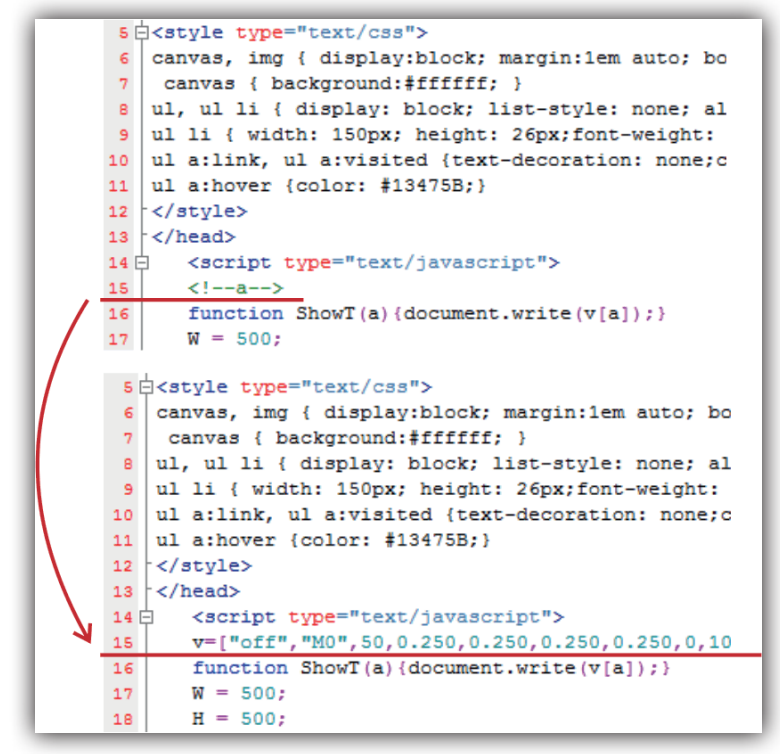

Fig. 10. Web page before (top) and after (bottom) replacing the SSI tag by the vector of current control parameters " $v$ "

accompanied by the data contents of the form. The Web server located on the controller executes a so-called Common Gateway Interface script (CGI script). That script parses the form data encoded in the Web page request and applies them to global variables, which control the behavior of the converter control algorithm. The user can apply subsequent changes of the con-

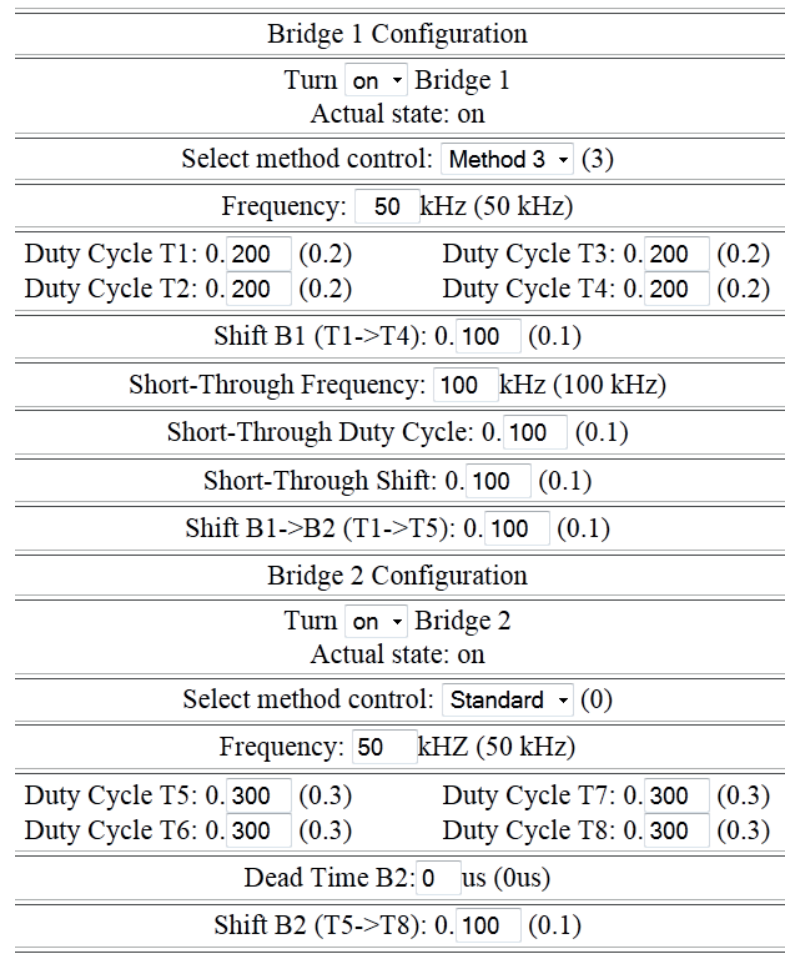

Fig. 11. Screenshot of the status Web page showing DAB settings used in the described experiment 


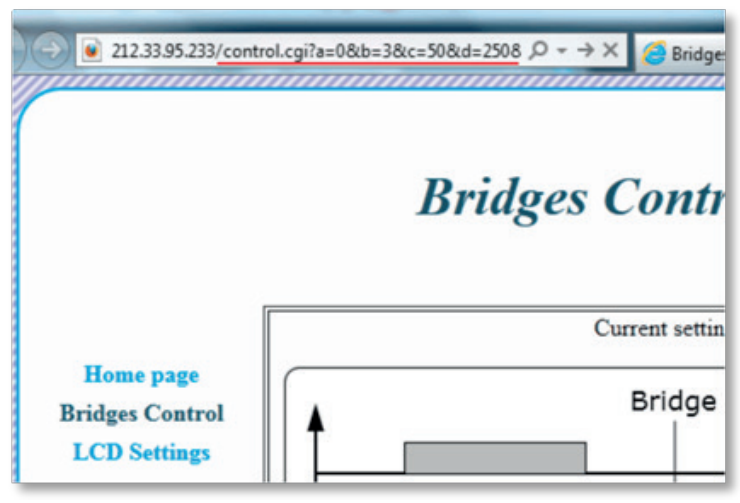

Fig. 12. Part of CGI-script-generated parameters that are sent to ARM by the POST method as part of the URL (red underline)

verter settings by repetitively committing the changes entered into the form shown in Fig. 11.

The form includes 21 different variables that are corresponding to various converter settings. When settings are changed, the JavaScript program is activated by the change and redraws the graphics inside the canvas. The changes are then committed by clicking or tapping the SUBMIT button located at the bottom of the Web page. The CGI script again verifies the settings against common errors that may occur, resulting in damage to the bridges. In the absence of any obvious problems, the settings are applied (Fig. 11).

a)

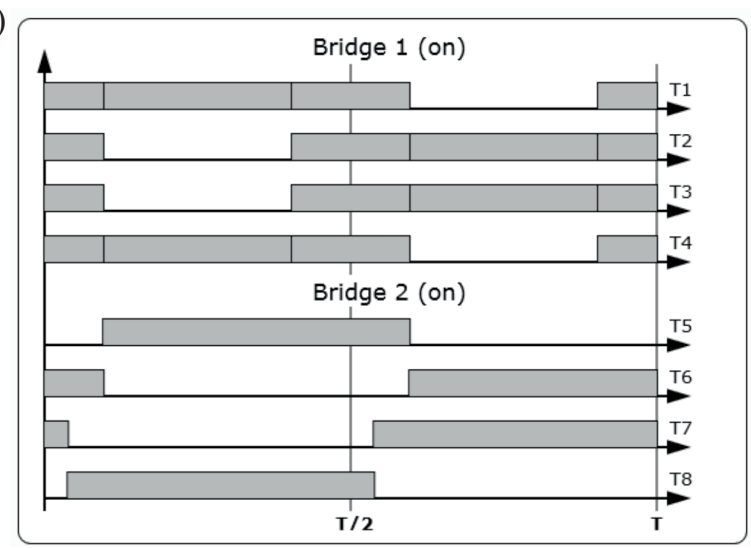

b)

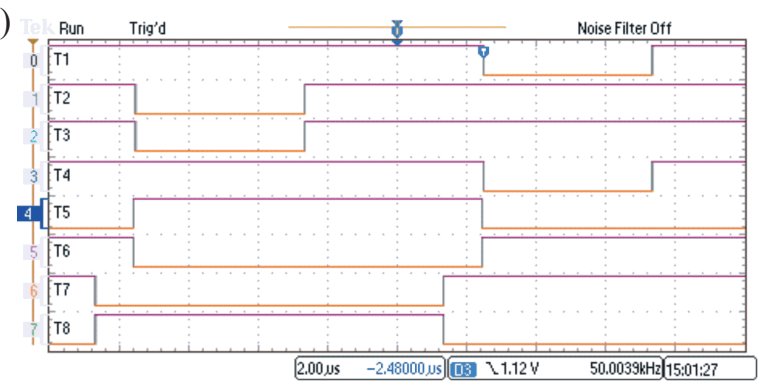

Then, the same Web page with the current (updated) settings is reloaded into the Web browser to allow for further control. This procedure helps minimize the likelihood of the user choosing improper settings, before applying them to the converter.

\section{System testing}

In order to test the performance of the converter and its Webbased user interface, several experiments were performed and the behavior of the converter was observed. The converter was set up in an open-loop circuit so that the output parameters need not be monitored directly. Fig. 12 shows a screen shot of the status Web page displaying the converter settings.

Optical output signals of the controller board were connected to inputs of the DAB converter. At the same time, oscilloscope probes were connected to "bridge A connector" and to "bridge B connector", as shown earlier in the top portion of Fig. 3, so that the output signals could be monitored and captured. Fig. 13 shows the signals observed during this experiment.

The converter settings, set using its Web interface, can be compared with the signals captured at the bridge connectors. Several different settings were used to verify whether the converter control system works properly. In all cases, the graphical representations of the transistor's control waveforms drawn on a Website (Fig. 13a, 13c) are consistent with the observed output signals (Fig. 13b, 13d).

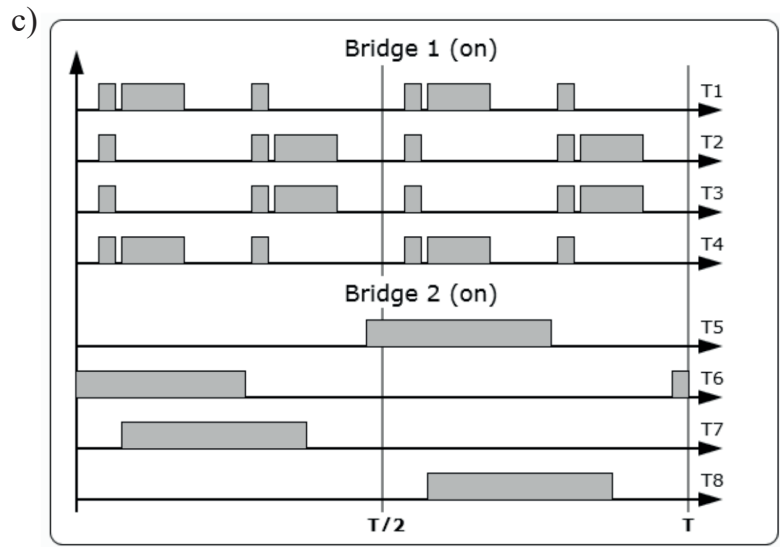

d)

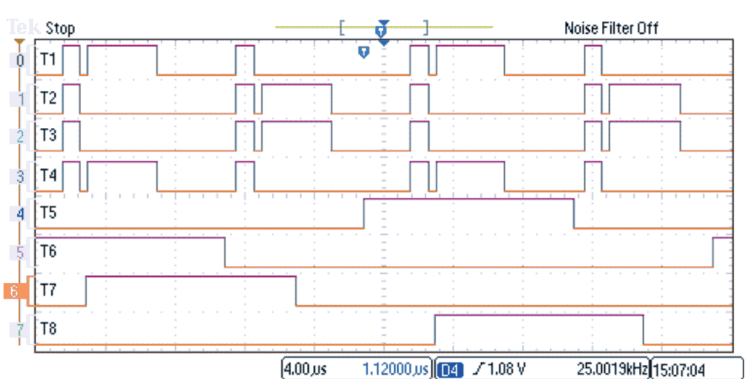

Fig. 13. Transistor control signals shown in web browser (a) and (c), as well as signals measured on the board control outputs (b) and (d) of the DAB converter 


\section{Conclusions}

This paper presented the design and successful test of a controller platform for a DAB converter. Multiple outputs allow its utilization not only in DAB but also in various other configurations of converters. Although the platform is inexpensive, it allows for the implementation of sophisticated control algorithms, for both local and remote user access, for monitoring interfaces, and for network communication using wired Ethernet and Internet Protocol set. The computational power of the system allows for further control algorithm expansion, as well as for increased security by the utilization of access authorization.

The experimental results on qZS-based DAB show that elaborated control board performs with high flexibility and simple operation of complicated power converters. The experiments show that besides changing the power flow direction, it is also possible to stabilize the DC-link voltage of either primary or secondary part of the converter. Besides current experiments, the developed control board provides the possibility to check new and complicated control algorithms for qZS-based DAB because of full flexibility to tune each output channel separately.

In the future, we foresee to achieve phase shift control of the qZS-based DAB that will allow the zero-voltage switching to be achieved, which will result in reduced switching losses and higher efficiency.

Also, from the safety point of view, safe operation zones can be established, and that creates the possibility to apply this system in education, or less-experienced personal users.

Acknowledgements. The work was supported by research project S/WE/3/2013 funded by The Scientific Subsidy of the Ministry of Science and Higher Education (MNiSW) and Latvian Council of Science (Grant 416/2012).

\section{REFERENCES}

[1] E.M. Natsheh, A.R. Natsheh, and A. Albarbar, "Intelligent controller for managing power flow within standalone hybrid power systems", IET Science, Measurement \& Technology 7 (4), 191-200 (2013).

[2] A. Malinowski, Y. Hao, "Comparison of embedded system design for industrial applications", IEEE Trans. on Industrial Informatics 7 (2), 244-254 (2011).

[3] B.M. Wilamowski and J.D. Irvin (Eds.), "Control and mechatronics", Sec. Ed. Taylor \& Francis, 683, 2011.

[4] L. Jian, H. Xue, G. Xu, X. Zhu, D. Zhao, and Z.Y. Shao, "Regulated hharging of plug-in hybrid electric vehicles for minimizing load variance in household smart microgrid", IEEE Trans. on Industrial Electronics 60 (8), 3218-3226 (2013).

[5] R.J. Kaplar, M.J. Marinella, S. DasGupta, M.A. Smith, S. Atcitty, M. Sun, and T. Palacios, "Characterization and reliability of SiC- and GaN-based power transistors for renewable energy applications", 2012 IEEE Energytech Conf., 29-31 (2012).

[6] H. Zhang, L.M. Tolbert, and B. Ozpineci, "Impact of SiC devices on hybrid electric and plug-in hybrid electric vehicles", IEEE Trans. on Industry Applications 47 (2), 912-921 (2011).
[7] M.P. Kazmierkowski, M. Jasinski and G. Wrona, "DSP-based control of grid-connected power converters operating under grid distortions", IEEE Trans. on Industrial Informatics 17 (2), 204-211 (2011).

[8] S.P. Engel, N. Soltau, H. Stagge, and R.W. De Doncker, "Dynamic and balanced control of three-phase high-power dual-active bridge DC-DC converters in DC-grid applications", IEEE Trans. on Power Electronics 28 (4), 1880-1889 (2013).

[9] A.J. Moradewicz and M.P. Kazmierkowski, "High efficiency contactless energy transfer system with power electronic resonant converter", Bull. Pol. Ac.: Tech. 57 (4), 375-381 (2009).

[10] A.J. Moradewicz and M.P. Kazmierkowski, "Contactless Energy Transfer System With FPGA-Controlled Resonant Converter”, IEEE Trans. on Industrial Electronics 57 (9), doi:10.1109/TIE.2010.2051395, 3181-3190 (2010).

[11] Web site, http://new.abb.com/smartgrids/what-is-a-smart-grid (2014).

[12] A. Espinoza, Y. Penya, J.C. Nieves, M. Ortega, A. Pena and D. Rodriguez, "Supporting Business Workflows in Smart Grids: An Intelligent Nodes-Based Approach", IEEE Trans. on Industrial Informatics 9 (3), 1384-1397 (2013).

[13] C. Cecati, G. Hancke, P. Palensky, P. Siano, and X. Yu, "Guest editorial special section on information technologies in smart grids", IEEE Trans. on Industrial Informatics 9 (3), 1380-1383 (2013).

[14] J. Berst, "Can the U.S. deploy an energy net the way it did the Internet?", SmartGridNews.com (28 Aug. 2012).

[15] R. Almeida, "Smart grid leverages ARM®-based solutions to enable intelligent power consumption with a more robust endto-end communication network", Texas Instruments, White papers, 1-8 (2012).

[16] R.U. Lenke, "A Contribution to the design of isolated DC-DC converters for utility applications", Ph.D dissertation, E.ON Energy Research Center, PGS Power Generation and Storage Systems, RWTH Aachen, 230, 2012.

[17] D. Aggeler, "Bidirectional galvanically isolated $25 \mathrm{~kW} 50 \mathrm{kHz}$ $5 \mathrm{kV} / 700 \mathrm{~V} \mathrm{Si-SiC}$ super cascode/Si-IGBT DC-DC converter", Ph.D. dissertation, ETH Zurich, 163, 2010.

[18] O. Husev, L. Liivik, F. Blaabjerg, A. Chub, D. Vinnikov, and I. Roasto, "Galvanically isolated quasi-Z-source DC-DC converter with a novel ZVS and ZCS technique", IEEE Trans. on Industrial Electronics 62 (12), 7547-7556 (2015).

[19] Web site, http://www.st.com/.

[20] Web site, http://www.arm.com/products/processors/cortex-m/.

[21] D. Vinnikov, I. Roasto, and J. Zakis, "New bi-directional DC/DC converter for supercapacitor interfacing in high-power applications", 14th International Power Electronics and Motion Control Conf. (EPE/PEMC) T11, 38-43, 6-8 (2010).

[22] V. Beldjajev, I. Roasto, and J. Zakis, "Impact of component losses of the efficiency of the new Quasi-Z source based dual active bridge", Technological Innovation for the Internet of Things: Doctoral Conference on Computing, Electrical and Industrial Systems. Portugal: Springer Heidelberg, 485-492 (2013).

[23] D. Vinnikov, I. Roasto; J. Zakis and R. Strzelecki: "New step-up DC/DC converter for fuel cell powered distributed generation systems: some design guidelines", in journal Electrical Review 86 (8), ISSN 0033-2097, 245-252 (2010).

[24] I. Roasto, D. Vinnikov, J. Zakis and O. Husev, "New shootthrough control methods for qZSI-Based DC/DC converters", IEEE Trans. on Industrial Informatics 9 (2), 640-647 (2013). 
[25] M.R. Palattella, N. Accettura, X. Vilajosana, T. Watteyne, L.A. Grieco, G. Boggia, and M. Dohler, "Standardized protocol stack for the Internet of (important) Things", IEEE Communications Surveys \& Tutorials 15 (3), 1389-1406 (2013).

[26] J.D. Decotignie, "The many faces of industrial ethernet [past and present]", IEEE Industrial Electronics Magazine 3 (1), 8-19 (2009).

[27] R.A.S. Fernandes, I.N. da Silva, and M. Oleskovicz, "Load profile identification interface for consumer online monitoring purposes in smart grids", IEEE Trans. on Industrial Informatics 9 (3), 1507-1517 (2013)
[28] N.M. Torrisi, "Monitoring services for industrial automation", IEEE Industrial Electronics Magazine 5 (1), 49-60 (2011).

[29] E. Marinoni, "RTOS, TCP/IP, File System, USB, CAN, etc.", http://www.emcu.it/STM32/RTOS-TCPIP-FileSystem-USB-etc/ RTOS-TCPIP-FileSystem-USB-etc.html (21 March 2015).

[30] Application Note 3966 - LwIP TCP/IP stack demonstration for STM32F407/STM32F417 microcontrollers, STMicroelectronics, 2011.

[31] Application Note 3969 - Secure socket layer (SSL) for STM32F417xx microcontroller, STMicroelectronics, 2011 